\title{
Sémiotique et anthropologie: un retour vers le futur?
}

\author{
Christelle de Oliveira-Verger \\ CeReS (Centre de Recherches Sémiotiques), Université de Limoges, France \\ christelle.de-oliveira@unilim.fr \\ Angelo di Caterino \\ CeReS (Centre de Recherches Sémiotiques), Université de Limoges, France \\ angelo.di@etu.unilim.fr
}

\begin{abstract}
Résumé
Cet article vise à rappeler les relations et le dialogue qu'entretiennent sémiotique et anthropologie depuis la théorie développée par Lévi-Strauss jusqu'au dialogue instauré récemment avec Descola. Nous nous attacherons à montrer comment interagissent ces deux disciplines, jusqu'à apparaître parfois telles les deux faces d'une même médaille. De plus, nous constaterons dans cette étude que les réflexions de Lévi-Strauss, Descola mais également de Geertz restent fondamentales pour des raisons qui confirment le lien entre anthropologie et sémiotique. La «bonne distance» ethnographique et la «bonne densité» analytique placeraient l'analyse entre Charybde et Scylla. La variété d'instruments à disposition servirait à éviter, d'un côté, le réductionnisme descriptif excessif à la faveur d'une stratification multi perspective et, de l'autre, elle réduirait le risque des dérives infinies au moyen d'une circonscription objectivante.
\end{abstract}

Mots clés: Sémiotique, anthropologie, identité, relation, schème conceptuel, point de vue.

\section{Semiotics and anthropology: a return to the future?}

\begin{abstract}
This article aims at recalling the relations and the dialogue that exists between semiotics and anthropology since the theory developed by Lévi-Strauss until the dialogue established recently with Descola. We will try to show how these two disciplines interact, until sometimes appearing at the two sides of the same coin. Moreover, it will be seen in this study that the reflections of LeviStrauss, Descola but also of Geertz remains fundamental for reasons that confirm the link between anthropology and semiotics. The ethnographic 'good distance' and the analytical 'good density' would place the analysis between Charybdis and Scylla. The variety of available instruments would serve to avoid, on the one hand, excessive descriptive reductions through multi-perspective stratification, and on the other, it would reduce the risk of infinite drift by means of an objectivizing circumscription.
\end{abstract}

Keywords: Semiotics, anthropology, identity, relationship, conceptual schema, point of view. 


\section{Introduction}

Comme le dit Klinkenberg ${ }^{1}$, à l'origine un sémioticien était un «agent-double» ${ }^{2}$ par la force des choses. Étant donné qu'il n'existait aucun cursus complet de sémioticien, celui-ci était forcément originaire et spécialiste d'une autre discipline, ce qui constituait un point d'ancrage afin de se diriger ensuite vers la science de la signification. Nous nous intéresserons ici plus particulièrement au parcours del'anthropologuesémioticien.

L'hypothèse globale qui guide cette étude est de mettre en évidence les relations et le dialogue qu'entretiennent sémiotique et anthropologie depuis la théorie développée par Lévi-Strauss jusqu'au dialogue instauré récemment avec Descola. Nous nous attacherons à montrer comment interagissent ces deux disciplines, jusqu'à apparaître parfois telles les deux faces d'une même médaille.

Nous établirons tout au long de ce travail un état de l'art des relations entre sémiotique et anthropologie, depuis Lévi-Strauss jusqu'à Descola, en passant par Geertz et nous observerons ces interactions au travers d'une analyse concrète sur un fait sociétal tel que la crise économique de 2008.

\section{Nouvelles tendances de la sémiotique au XXI ${ }^{\mathrm{e}}$ siècle: la question des "pratiques».}

Historiquement, l'évolution théorique de la sémiotique a coïncidé avec les défis analytiques d'objets de plus en plus complexes. En suivant cette direction et en résolvant de plus en plus de difficultés, la sémiotique a lentement abandonné le texte, en tant qu'objet d'étude privilégié, et a envisagé le besoin de sémiotiques objectales. L'ancienne et «archaïque» sémiotique greimasienne semble en effet n'être plus en mesure de pouvoir dire «quelque chose de sensé sur le sens» par rapport à des objets dont la nature phénoménale manifeste la nécessité de nouveaux instruments analytiques. Dans la dernière décennie, la question des pratiques vu leur complexité, leur nature continue et non discrétisable et le fait qu'elles soient aussi des textes non clôturables contrairement à d'autres systèmes de signification - a hanté le sommeil des sémioticiens.

Le travail de Jacques Fontanille ${ }^{3}$ a relevé ce défi de grande portée en résumant en premier lieu, mais surtout en systématisant, la réflexion théorique et analytique entre-temps élaborée sur les pratiques en sémiotique. Or, outre la portée euristique du texte de Fontanille, nous désirons souligner que, de fait, la recherche sur les pratiques redonne à la sémiotique sa place parmi les sciences sociales, en revalorisant le dialogue, déjà en cours et à certains égards nécessaire, avec les disciplines ethno-anthropologiques. Il s'agit d'un dialogue qui se manifeste presque explicitement dans les pages de Fontanille et qui, de fait, jette un pont théorique, souhaité par Greimas même, avec la pensée de Pierre Bourdieu.

Actuellement, suite aux premiers travaux de Fontanille et à leur évolution ont fait suite, outre les innombrables journées d'étude, des colloques, des séminaires et des articles. La parution récente de deux ouvrages, signés l'un par Fontanille ${ }^{5}$ et l'autre par Fontanille et Couégnas $^{6}$, relance concrètement la possibilité d'une anthropo-sémiotique qu'il faudrait

1 Jean-Marie Klinkenberg, "Ce que la sémiotique fait à la société, et inversement», Signata. Annales des sémiotiques / Annals of Semiotics, 31 décembre 2012, no 3, pp. 13-25.

2 Paolo Fabbri, «Nous sommes tous des agents doubles», in, Edition du Seuil., Paris, 1988, pp. 325-341.

3 Jacques Fontanille, Pratiques sémiotiques, 1re éd., Paris, Presses universitaires de France, coll. «Formes sémiotiques», 2008.

4 Jacques Fontanille, au cours de son intervention au Congrès des Jeunes Chercheurs en sémiotique a rappelé la volonté irréalisée de Greimas de consacrer un numéro des Actes sémiotiques à se confronter avec l'anthropologie de Bourdieu.

5 Jacques Fontanille, Formes de vie, Liège, Presses universitaires de Liège, 2015.

6 Jacques Fontanille et Nicolas Couegnas, Terres de sens, 2017. 
probablement rapprocher de l'idée d'ethnosémiotique déjà élaborée par Greimas dans son dictionnaire ${ }^{7}$. Cette tendance, qui s'apparente actuellement à une véritable vague, a identifié, entre-temps, de nouveaux et importants interlocuteurs parmi les ethno-anthropologues. Parmi eux, nous rencontrons des noms éminents comme celui de Bruno Latour ${ }^{8}$ ainsi que ceux de quelques représentants du tournant ontologique en anthropologie, comme Philippe Descola et Viveiros de Castro.

\section{Une actualité venant du passé.}

Avant d'examiner par un exemple analytique la portée de ce dialogue contemporain, nous aimerions faire rapidement le point sur cette entente entre anthropologie et sémiotique. En effet, il convient de rappeler que la sémiotique, en tant que théorie standard développée par Greimas et ses collaborateurs, contient l'influence énorme de l'anthropologie structurale de Lévi-Strauss. Si d'un côté la présence de cette contamination est parfois reconnue par les chercheurs les plus experts, de l'autre côté on a pris rarement en charge la possibilité d'une coïncidence entre les deux disciplines ce qui, par ailleurs, légitimerait le dialogue contemporain en cours.

Il ne faut pas oublier que, déjà en 1960, LéviStrauss, lors d'une situation institutionnellement importante comme la conférence inaugurale $\mathrm{du}$ cours d'anthropologie sociale au Collège de France, définit de la même manière l'anthropologie sociale en l'identifiant au le projet de sémiologie de Ferdinand de Saussure:

Qu'est-ce donc que l'anthropologie sociale? Nul, me semble-t-il, n'a été plus près de la définir - bien que ce soit par prétérition - que
Ferdinand de Saussure, quand, présentant la linguistique comme une partie d'une science encore à naître, il réserve à celle-ci le nom de sémiologie, et lui attribue pour objet d'étude la vie des signes au sein de la vie sociale?

Lévi-Strauss caractérise la sémiologie comme un cadre de repère unifiant toutes les recherches sur les systèmes de signification. Il s'agit d'un domainequisemblebien défini pourLévi-Strauss, mais très peu occupé, de sorte qu'il affirme qu'en l'absence de prétendant, l'anthropologie est la discipline qui détient le droit de s'occuper de cette science future imaginée par Saussure et, comme on le sait, développée ensuite par Greimas ${ }^{10}$ à l'aide des apports essentiels de la glossématique de Hjelmslev ${ }^{11}$. Autrement dit, il est possible d'envisager que le projet sémiotique de l'école de Paris a été, et probablement l'est encore, un synonyme du projet anthropologique structural. De fait, par une revalorisation historique sur la naissance des instruments analytiques mise au point par Greimas et par ses élèves, on retrouve le rapport incessant que ces instruments théoriques ont gardé par rapport à la réélaboration structurale de l'anthropologie lévistraussienne.

\section{Sémiotique et Anthropologie: une systématisation.}

L'affirmation précédente de Lévi-Strauss nous pose une question intéressante sur laquelle il faut s'interroger aujourd'hui, face aux nouvelles tendances. C'est à dire que, parmi les sciences sociales, le rôle de la sémiotique estil celui d'une anthropologie? Pour répondre à cette question, qui à certains égards peut sembler risquée et insensée et essayer d'établir clairement la construction d'une hiérarchie entre les disciplines, il faut examiner soigneusement

$7 \mathrm{Il}$ faut rappeler que le projet d'Ethno-sémiotique contenant des références anthropologiques explicites, a déjà été développé par la sémiotique italienne. Il trouve sa systématisation dans les travaux de Francesco Marsciani. 8 Voir le dossier Sémiotique et anthropologie des modernes qui vient de paraître dans la rubrique «Projets et programmes» des Actes Sémiotiques.

9 Claude Lévi-Strauss, Anthropologie Structurale 2, Paris, Plon, p. 18.

10 Algirdas Julien Greimas, Du sens I: essais sémiotiques, Paris, Ed. du Seuil, 2012.

11 Louis Hjelmslev, Prolégomènes à une théorie du langage suivi de La structure fondamentale du langage, Nouvelle édition., Paris, Éditions de Minuit, coll. «Arguments», n 35, 1971. 
quelques idées de Lévi-Strauss ${ }^{12}$, reprises par Descola $^{13}$, quant aux rapports entre ethnographie, ethnologie et anthropologie.

Dans une espèce de structure englobant ethnographie, ethnologie et anthropologie, le point de vue commun à ces deux géants de l'anthropologie française est de se partager, à différents niveaux d'abstraction, de recherche sur l'étude de la culture, un objet qui dans ses innombrables définitions semble prendre l'aspect du sens ou de la signification pour les sémioticiens.

L'ethnographie correspond aux premiers stades de la recherche, c'est l'enquête sur le terrain et la collecte de données, aboutissant ordinairement à une étude ou description monographique, circonscrite dans le temps et dans l'espace.

L'ethnologie prolonge l'ethnographie et désigne un effort de synthèse visant à des généralisations suffisamment vastes à un niveau régional (élargissement $\mathrm{du}$ corpus d'analyse) ou thématique (attention portée sur un type de phénomène ou de pratique) pour que le recours à des sources ethnographiques secondaires en constitue le préalable obligé et la mise au jour de propriétés comparables, le résultat attendu.

L'anthropologie représente le dernier moment de la synthèse: sur la base des enseignements de l'ethnographie et de l'ethnologie, elle aspire à produire une connaissance théorique globale de l'homme, en découvrant les principes qui rendent intelligible la diversité de ses productions sociales et de ses représentations culturelles au long des siècles et à travers les continents.

En un sens, il serait intéressant de faire coïncider cette triade avec la stratification imaginée par Greimas quant à une sémiotique descriptive, méthodologique et épistémique ${ }^{14}$.

Cependant, le rôle exact que Lévi-Strauss et Descola attribuent à l'anthropologie semble pouvoir ou devoir être joué par la sémiotique. $\mathrm{Au}$ fond, la sémiotique n'est-elle qu'une théorie générale de la signification capable d'annuler toutes les différences expressives des divers textes -qui ne sont que des représentations culturelles- en fournissant, en plus des descriptions ethnographiques et des généralisations ethnologiques, un ensemble d'instruments analytiques?

Nous allons explorer certaines possibilités qu'ouvre ce questionnement au travers de l'étude d'un cas concret qui explique le croisement contemporain de l'actualité avec le modèle de Descola.

\section{Sémiotique et anthropologie: modes d'identification et modes de relation}

L'exemple que nous allons traiter maintenant trouve son origine au cours de l'été 2007 lorsque le marché américain des prêts immobiliers hypothécaires, les prêts subprimes, s'effondre en engendrant une crise violente qui, en quelques mois, s'étend au monde entier et frappe divers secteurs de la finance jusqu'à atteindre l'économie réelle. Ce travail se situe dans un cadre épistémologique où les frontières entre sémiotique et anthropologie deviennent perméables les unes aux autres. Il permet d'analyser comment la théorie sémiotique interagit avec la théorie anthropologique concernant les modes d'identification et les modes de relation élaborés par Descola ${ }^{15}$. La sémiotique, par l'intermédiaire d'outils théoriques efficaces, propose ainsi un éclairage

12 Cf. Claude Lévi-Strauss, Anthropologie Structurale, Paris, Plon, chap. I.

13 Cf. Philippe Descola, «Claude Lévi-Strauss, une présentation», en La lettre du College de France, Hors Série, Novembre 2008, pp. 4-9.

14 Cf. Algirdas Julien Greimas \& Joseph Courtes, Sémiotique. Dictionnaire raisonné de la théorie du langage, Paris, Hachette, 1979, pp. 224-226.

15 Philippe Descola, Par-delà nature et culture, Paris, Gallimard, coll. «Bibliothèque des sciences humaines», 2005. 
nouveau et nécessaire au déploiement d'une réflexion ouverte et originale sur des questions d'intérêt social, comme la crise financière. En préalable à notre analyse, nous allons donc exposer rapidement les grands principes de cette théorie anthropologique.

Chaque société est considérée comme un ensemble signifiant et, à ce degré de généralité, les sémioticiens et les anthropologues définissent pareillement une société par la façon dont elle pose la frontière entre soi et autrui. Définir une frontière revient simultanément à accepter de ressembler à l'autre, mais également à cibler ce qui permet de s'en différencier. Descola ${ }^{16}$ pose ainsi l'hypothèse que les schèmes intégrateurs des pratiques peuvent être ramenés à deux modalités fondamentales de structuration de l'expérience individuelle et collective. Il les nomme: l'identification et la relation. Pour lui, il est nécessaire de concevoir séparément ces deux modes d'intégration d'autrui qui distinguent jugement d'inhérence et jugement de relation.

Il envisage la relation comme des rapports externes entre des êtres et des choses repérables dans des comportements typiques. Ceux-ci sont susceptibles de faire l'objet d'une traduction partielle dans des normes sociales concrètes. Ainsi, les relations qu'il étudie sont celles qui se dégagent de pratiques observables et non celles qui pourraient être déduites de règles formelles gouvernant les propositions logiques. Il définit la réciprocité comme étant un mode d'identification à l'autre qui établit le minimum de la reconnaissance mutuelle du semblable.

Des mécanismes de propagation interviennent ensuite car, lors d'une rencontre avec certaines unités sociales, il existe des acteurs ou groupes avec lesquels des limites sont atteintes, à ce moment-là cette réciprocité peut ne plus fonctionner ${ }^{17}$. Si Lotman ${ }^{18}$ définissait le principe de différence «au sein de» la sémiosphère par l'opposition «nous» / «autrui», Descola ${ }^{19}$ tient le même raisonnement et se pose également le problème en ces termes: qui est soi-même qui est autrui? Comment se déroule l'opération d'agglomération des relations mutuelles et réciproques? Quels sont les processus de différenciation avec ce qui n'est pas soi?

Les modes de relations apparaissent comme des schèmes intégrateurs qui relèvent d'un certain type de structures cognitives, émotionnelles et sensori-motrices. Cellesci canalisent la production d'inférences automatiques, orientent l'action pratique et organisent l'expression de la pensée et des affects selon des trames plus ou moins stéréotypées. Contrairement au mode d'identification, un mode de relation dominant se singularise en exprimant un écart très important avec les styles $\mathrm{d}$ 'interactions et de comportements quotidiens en vigueur dans le voisinage immédiat. Comme si chaque collectif humain tenait prioritairement à se distinguer de la manière la plus efficace possible des collectifs humains alentour avec lesquels il coexiste.

Descola accorde donc une prédominance aux modes d'identification sur les modes de relation ${ }^{20}$. Les modes d'identification spécifient les propriétés ontologiques des termes et orientent en partie la nature des relations pouvant les unir.

Il distingue quatre modes d'identification à disposition des humains vivants en société, mais il y inclut également les catégories des non-humains relevant des animaux et des plantes ainsi que les relations sociétales qu'ils sont susceptibles d'entretenir les uns avec les autres.

16 Ibid. p. 204

17 Marcel Mauss, Essai sur le don: forme et raison de l'échange dans les sociétés archaïques, Paris, Presses universitaires de France, 2007. Mauss explique ces mécanismes de propagation à l'intérieur du chapitre II et III p 131 à 169. 18 Yuri Lotman, La sémiosphère, Limoges, PULIM, coll. «Nouveaux actes sémiotiques», 1999.

19 Philippe Descola, Par-delà nature et culture, op. cit.

20 Ibid. 
La société animiste dans laquelle le monde spirituel est général aux humains, aux plantes et aux animaux alors que le monde physique va distinguer ces différents ordres du vivant.

Le totémisme se caractérise comme une société où les équivalences sont totales. On y observe la création d'alliances entre des clans humains et des espèces animales ou végétales qui se mesurent à d'autres alliances créées de la même manière. Chaque alliance ainsi constituée adopte le totem comme mode de figuration.

Les sociétés naturalistes correspondent au modèle des sociétés occidentales dans lesquelles nous vivons. À l'intérieur de ces sociétés naturalistes, la notion de culture est nécessaire. Ce qui la caractérise est l'idée que le végétal, l'animal et l'humanité sont différents selon le monde spirituel, mais obéissent aux mêmes lois biologiques de la nature quant à leurs enveloppes corporelles.

Tout est différent dans la société analogique. La différenciation est présente sur tous les plans, aussi bien dans le physique que dans le spirituel. Ainsi, pour pouvoir se reconnaitre comme appartenant à la même société, tout en se distinguant des autres, ils définissent des catégories différentes par analogie et établissent des liens qui permettent de constituer des ensembles caractérisés comme des sociétés.

Descola retient ensuite un ensemble de six modes de relation qui jouent un rôle essentiel dans les rapports entre humains et non humains. Ils viennent moduler les quatre modes d'identification décrits précédemment. Ils se nomment: l'échange, la prédation, le don, la production, la protection et la transmission. Il est possible de les scinder en deux sous-groupes selon le type de relation entretenu entre les différents termes.

Le premier comprend l'échange, la prédation et le don et se détermine par des relations potentiellement réversibles entre des termes qui se ressemblent. Le second est constitué de la production, la protection et la transmission et se caractérise par des relations univoques fondées sur la connexité entre des termes non équivalents.
L'échange se définit comme une relation symétrique dans laquelle tout transfert volontaire d'une entité à une autre nécessite une contrepartie.

La prédation et le don sont, quant à eux, des relations asymétriques. Dans le cas de la prédation, c'est une asymétrie négative c'est-àdire qu'une entité prend une valeur à une autre entité sans lui offrir de contrepartie. En ce qui concerne le don, c'est une asymétrie positive dans laquelle une entité offre une valeur à une autre entité sans en attendre de compensation. Il établit des modes d'identification qui orientent en partie le mode de relation sous-jacent, mais sans pour autant le déterminer.

5. Sémiotique et anthropologie, une application concrète: le rôle de l'actionnaire dans la crise financière

Jusqu'à la fin des années 1970, l'actionnaire était, dans les entreprises, le dernier maillon de la chaine en ce qui concerne la distribution des profits comme il est prévu dans le pacte entrepreneurial du modèle fordien. Celui-ci prônait un équilibre primordial entre l'entreprise et ses salariés et l'actionnaire. Les fruits de la croissance économique étaient directement répercutés sur le salaire des ouvriers, qui augmentait régulièrement, et sur l'amélioration de leur protection sociale. De cette manière, ils étaient à la source d'une consommation forte qui conduisait à une croissance qui reposait sur des bases concrètes et quantifiables. Ainsi, à l'intérieur de nos sociétés occidentales d'aprèsguerre, le profit matériel et financier de l'individu est corrélé avec une stabilisation de l'ordre social ainsi qu'avec le fonctionnement harmonieux $\mathrm{du}$ système économique.

Pour comprendre la logique des multiples flux qui ne cessent jamais d'avoir lieu entre ces différents agents, encore faut-il rappeler que chacun de ces flux engage une opération de confiance. Sans elle, l'échange, le prêt, etc., ne pourraient avoir lieu: la sociologie durkheimienne de François Simiand à Marcel Mauss, nous incite justement, à rapprocher la «circulation monétaire» des relations d'interdépendance sociale rendues possible 
par une croyance collective commune, une solidarité préalable, et, plus précisément, par la «confiance mutuelle minimale» qu'elles engendrent entre les différents agents qui «contractent $»^{21}$.

Il est donc indispensable que les choix opérés par les différents acteurs du système dans les actes ordinaires de leur existence soient dans leur intérêt, en fonction de leurs attentes et de leurs ressources, mais soient aussi cohérents avec l'intérêt du système économique dans son ensemble. Ces choix exercent un pouvoir d'orientation du processus de l'échange et, l'utilisation de croyances économiques sert de référence pour rationaliser ses désirs personnels face à intérêt général de la collectivité.

Ainsi, dans le système économique occidental, le mode de relation de l'échange, caractéristique du naturalisme, est dominant et associé au mode de relation de la production. La production désigne une relation que les hommes établissent entre eux selon des formes définies afin de se procurer, ensemble, des moyens d'existence.

L'idée est devenue commune que l'histoire de l'humanité est avant tout fondée sur le dynamisme introduit par la succession des manières de produire des valeurs d'usage et des valeurs d'échange à partir des matériaux que l'environnement fournit ${ }^{22}$.

Cependant, au début des années 1980, certaines personnalités politiques anglosaxonnes, notamment Ronald Reagan et Margaret Thatcher ont entrepris de mener des réformes économiques sur ce pacte. À partir de ce moment-là, les «stock-options» ou «options sur actions» en français représentent la part la plus importante de la rémunération de l'actionnaire. Elles reposent sur le droit gratuit qu'a le bénéficiaire de ces options, d'acheter un certain nombre d'actions de la société par projection à cinq ou dix ans au prix actuel.
Ces mesures ont une conséquence sur le rôle et le statut des actionnaires qui ne sont plus obligés de choisir une entreprise dont il voulait accompagner le développement en investissant régulièrement des fonds pendant un ou dix ans. À présent leur rôle tend à se confondre avec celui d'un spéculateur ayant la possibilité de rester propriétaire d'une action une semaine, voire moins d'une heure ou d'une minute. C'est ainsi que ces nouvelles structures actionnariales attendent des entreprises que celles-ci aient pour objectif principal la rentabilité. Ceci a pour conséquence de détourner le capitalisme des projets industriels car gagner de l'argent n'est plus la conséquence qui récompense des projets, mais devient le seul but. Ce système pénalise l'investissement puisque l'actionnaire ne reste plus dans l'entreprise. Il s'ensuit une course à la rentabilité que poursuivront les multinationales jusqu'à la crise: le record d'une année devenant la norme de l'année suivante.

C'est bien la prédation du point de vue des valeurs, au sens où l'entend Descola, qui prend le pas sur l'échange et la production. Il désigne le contraire du don sous le terme de prédation c'està-dire le fait s'emparer d'une chose sans offrir de contrepartie. C'est un acte qui n'engendre pas d'obligation pour celui qui s'y livre et qui est envisagé dans le sens d'une contrainte fondamentale inhérente à la vie animale et caractéristique d'une société animiste.

La prédation est donc un phénomène de destruction productive indispensable à la perpétuation d'un individu; loin d'exprimer une cruauté gratuite ou un désire pervers d'anéantissement, elle transforme au contraire la proie en un objet de la plus haute importance pour celui qui l'incorpore: la condition même de sa survie ${ }^{23}$.

La prédation apparait ainsi, non comme une manifestation débridée de férocité érigée en vertu collective, mais comme une disposition à

21 Frédéric Lebaron, La crise de la croyance économique, Editions du croquant, coll. «dynamiques socioéconomiques», 2010.

22 Philippe Descola, Par-delà nature et culture, op. cit.

23 Ibid. p. 543. 
incorporer une altérité humaine ou non humaine au motif qu'elle s'avère indispensable à la définition du soi. Dans une perspective anthropologique qu'il est nécessaire de transposer dans une perspective économique et financière, il définit une attitude prédatrice à autrui comme étant:

la nécessité ressentie d'incorporer sans cesse les corps et les identités des voisins afin de persister à être soi-même tout en étant déterminé en partie par ce que l'on capture et assimile, la dénégation têtue de la réciprocité librement assumée ${ }^{24}$.

L'attitude des actionnaires privilégiant le profit et la rentabilité des actions à court terme, plutôt qu'une bonne gestion concrète d'une entreprise sur le long terme est ainsi caractéristique du mode de relation de la prédation. Ce mode de relation interférant avec celui de la production et de l'échange, garants du bon fonctionnement $\mathrm{du}$ système économique, et prenant de plus en plus d'ampleur est ainsi à l'origine de dysfonctionnements ayant mené à la crise financière qui a ébranlé le monde en 2008.

La vision de la sémiotique comme anthropologie était presque inimaginable jusqu'à hier. Aujourd'hui on essaie de systématiser cette fusion qui semble légitime. Ainsi, la recherche actuelle est concentrée à la découverte d'autres anthropologues qui, pour des raisons différentes, revalorisent l'importance de la sémiotique dans leur domaine de recherche.

\section{Geertz et la sémiotique comme «instance de contrôle».}

Pour conclure sur la nécessité du dialogue entre anthropologie et sémiotique nous voulons souligner l'influence de Clifford Geertz, dont les travaux n'ont connu qu'une faible diffusion en France. Il souligne l'importance de la sémiotique comme seule interlocutrice pour la solution d'un problème analytique qui concerne une variété infinie d'interprétations se succédant sur les mêmes objets. Dans l'introduction d'un de ses travaux les plus importants, Geertz expose son idée d'anthropologie. Il écrit que:

Le concept de culture auquel j'adhère [...] est essentiellement sémiotique. Croyant $[. .$.$] que$ l'homme est un animal pris dans les réseaux de signifiance qu'il a lui-même tissés, je considère la culture comme assimilable à ces réseaux, et par suite son analyse comme relevant non d'une science expérimentale en quête de loi, mais d'une science interprétative en quête de sens ${ }^{25}$.

Pour paraphraser, selon Geertz l'homme est naturellement plongé à l'intérieur de la culture, un monde qui a déjà du sens et que lui même a créé ${ }^{26}$. Les spécificités naturelles de l'homme seraient d'apprendre, produire et transmettre de la culture ${ }^{27}$.

Même si, dans une perspective refusant l'identification lévistraussienneentre sémiotique, anthropologie et structuralisme, Geertz parvient à des compromis très intéressants comme par exemple que chaque phénomène culturel est un système de signes (ou de symboles). Les phénomènes culturels à analyser, les textes, apparaissent comme des micro-représentations de la culture, c'est-à-dire le moyen par lequel elle se manifeste et se communique à l'intérieur de la vie sociale. En ce sens, une sémiotique devrait se consacrer à ces véhicules de signifié qui se transmettent dans le réseau de la culture.

Si nous devons avoir une sémiotique de l'art (aussi bien que de tout système de signes [...]), nous allons devoir nous engager dans une sorte $[\ldots]$ d'ethnographie des véhicules de transmission du sens. De tels signes et symboles, de tels véhicules du sens, jouent un

$24 \mathrm{Ibid}, \mathrm{p} .582$.

25 Clifford Geertz, The Interpretation of Cultures, New York, Basic Books, 1973, p. 5 (notre traduction).

26 Il s'agit d'une idée identique à celle de Greimas concernant la notion de «monde naturel».

27 Cf. Clifford Geertz, The Interpretation of Cultures, New York, Basic Books, 1973, pp. 43-51. 
rôle dans la vie d'une société [...] et c'est cela en fait qui leur donne leur vie. [...] Le sens est l'usage [...] ou plus soigneusement le résulte de l'usage ${ }^{28}$.

\section{Conclusion}

En conclusion, comme nous avons pu le constater dans cette étude, les réflexions de LéviStrauss, Descola mais également de Geertz restent fondamentales pour des raisons qui confirment le lien entre anthropologie et sémiotique. La «bonne distance» ethnographique et la «bonne densité» analytique placeraient l'analyse entre Charybde et Scylla. La variété d'instruments à disposition servirait à éviter, d'un côté, le réductionnisme descriptif excessif à la faveur d'une stratification multi perspective et, de l'autre, elle réduirait le risque des dérives infinies au moyen d'une circonscription objectivante.

En définitive, la demande implicite que Geertz adresse à la sémiotique est celle d'une instance de contrôle, c'est à dire d'un domaine théorique qui permettrait de parler d'une véritable science anthropologique. Malgré l'espace théorique énorme qui sépare Geertz de Descola et LéviStrauss, nous retrouvons la proposition de l'anthropologue français qui prévoyait que dans la tripartition entre ethnographie-ethnologie et anthropologie, la sémiotique jouerait le rôle de cette dernière ou coïnciderait avec elle.

\section{Bibliographie}

Descola Philippe, (2008) «Claude Lévi-Strauss, une présentation», in, La lettre du Collège de France, Hors-Série, novembre.

Descola Philippe, (2005). Par-delà nature et culture, Paris, Gallimard, coll. «Bibliothèque des sciences humaines».

Fabbri Paolo, (1988) «Nous sommes tous des agents doubles», in, Edition du Seuil., Paris, pp. 325-341.
Fontanille Jacques, (2015) «La sémiotique face aux grands défis sociétaux du XXIe siècle», AS - Actes Sémiotiques, 30 janvier, no118.

Fontanille Jacques (2015). Formes de vie. Liège: Presses universitaires de Liège, collection «Sigilla», n 3 .

Fontanille Jacques (2008). Pratiques sémiotiques, Paris, Presses universitaires de France, collection «Formes sémiotiques».

Fontanille Jacques et Couégnas Nicolas. (2017). Terres de sens (à paraître).

Geertz Clifford. (1973). The Interpretation of Cultures. New York: Basic Books.

Geetz Clifford. (1983). Local Knowledge, Further Essays in Interpretive Anthropology, New York: Basic Books.

Greimas Algirdas Julien. (2012). Du sens [I]: essais sémiotiques, Paris, Edition du Seuil.

Algirdas Julien et Courtes Joseph (1993). Sémiotique: dictionnaire raisonné de la théorie $d u$ langage. Paris, Hachette supérieur, collection «Hachette université».

Hjelmslev Louis (1971). Prolégomènes à une théorie du langage suivi de La structure fondamentale du langage, Nouvelle édition, Paris : Éditions de Minuit, collection «Arguments», $\mathrm{n}^{\circ} 35$.

Klinkenberg Jean-Marie (2012) «Ce que la sémiotique fait à la société, et inversement», Signata. Annales des sémiotiques / Annals of Semiotics, 31 décembre, no 3, pp. 13-25.

Latour Bruno (2012). Enquête sur les modes d'existence: une anthropologie des modernes, Paris: La Découverte, 498 p. 
Lebaron Frédéric (2000). La croyance économique: les économistes entre science et politique, Paris, Seuil, coll. «Liber».

Lévi-Strauss Claude (1958). Anthropologie structurale, Paris: Plon.

Lévi-Strauss Claude (1973). Anthropologie structurale 2, Paris: Plon.

Lotman Yuri (1999). La Sémiosphère, Limoges, PULIM, coll. «Nouveaux actes sémiotiques».

Marsciani Francesco (2007). Tracciati di etnosemiotica, Milan, Franco Angeli.

Marsciani Francesco (2014). «À propos de quelques questions inactuelles en théorie de la signification», AS - Actes Sémiotiques, 30 juin, no 117.

Mauss Marcel (2007). Essai sur le don: forme et raison de l'échange dans les sociétés archaïques. Paris, Presses universitaires de France, coll. «Quadrige» 